Pacific Journal of Mathematics

ON LOCAL CONVEXITY OF BOUNDED WEAK TOPOLOGIES 


\title{
ON LOCAL CONVEXITY \\ OF BOUNDED WEAK TOPOLOGIES \\ ON BANACH SPACES
}

\author{
J. GoMEZ GIL
}

In this paper we prove that the bw topology on a Banach space $E$, i.e. the finest topology which agrees with the weak topology on bounded sets of $E$, is a locally convex topology if and only if the Banach space $E$ is reflexive.

1. Introduction. If $E$ is a Banach space, the bounded weak (bw) topology is the finest topology which agrees with the weak topology on bounded sets. Wheeler in [7, p. 251] proves that the bw topology on $c_{0}$ is not locally convex. This result gives a counterexample to a remark of Day [2, p. 42] which said that the bw topology is locally convex always. This fact suggests a question: Under what conditions on $E$ is it true that bw is a locally convex topology?. The theorem of Banach and Dieudonné (2.2) shows that reflexivity is a sufficient condition. In this paper we obtain that reflexivity is also a necessary condition.

2. Notations, definitions and preliminary results. The notations for topological vector spaces are taken primarily from [6], but we employ the definition of polarity found in [4].

Definition 2.1. If $E$ is a locally convex space (lcs), the equicontinuous weak* (ew*) topology on $E^{\prime}$ is the finest topology on $E^{\prime}$ which coincides with the weak* topology $\sigma\left(E^{\prime}, E\right)$ on equicontinuous sets of $E^{\prime}$.

The following result characterizes this topology when $E$ is a metrizable lcs.

THEOREM 2.2. (Banach-Dieudonné.) Let $E$ be a metrizable locally convex space and $E^{\prime}$ its dual. The ew* topology on $E^{\prime}$ is the topology of the uniform convergence on precompact subsets of $E$.

For a demonstration of this theorem we refer the reader to [4] or [6].

As an immediate consequence of this theorem we have that if $E$ is a metrizable lcs, the ew* topology on $E^{\prime}$ is locally convex. 
Other results about ew* may be found in [1] and [2].

Definition 2.3. If $E$ is a locally convex space, the bounded weak (bw) topology on $E$ is the finest topology on $E$ which agrees with the weak topology $\sigma\left(E, E^{\prime}\right)$ on bounded sets.

This definition is equivalent to Day's [2, p. 41]:

"The bw topology is the collection of all subsets $U$ of $E$ satisfying: for each bounded set $B$ of $E$, there is a $\sigma\left(E, E^{\prime}\right)$-open $V$ with $U \cap B=V \cap$ B."

Obviously the last definition is not changed if we choose the bounded sets on a fundamental family of bounded sets in $E$.

As follows from [1, p. 265], the bw topology is semi-linear, i.e. addition and scalar multiplication functions are separately continuous. Moreover, if $E$ is a Banach space, it can be shown [3, p. 21] that bw is a vectorial topology if and only if it is a locally convex one.

A general result of Collins [1, p. 266], which can be extended to the complex case, makes the following definition valid.

Definition 2.4. The convex bw (cbw) topology on a locally convex space $E$ is the unique locally convex topology with a base of all convex neighborhoods of 0 in the bw topology.

It is easy to see that the cbw topology is the finest locally convex topology which agrees with the weak topology on bounded sets. In [7, p. 251] may be found the following result which characterizes the cbw topology:

THEOREM 2.5. If $E$ is a lcs, the cbw topology on $E$ is that of uniform convergence on compact subsets of the completion of $\left(E^{\prime}, \beta\left(E^{\prime}, E\right)\right)$.

As consequence of this result and Theorem 2.2 we obtain:

COROllary 2.6. If $E$ is a Banach space, the cbw topology is the restriction to $E$ of the ew* topology on $E^{\prime \prime}$.

In particular if $E$ is reflexive we have:

COROLlaRy 2.7. If $E$ is a reflexive Banach space, the bw topology on $E$ is a locally convex topology. 
A different introduction and other results about this topology may be seen in [3].

If $E$ is a Banach space, we denote by $B, B^{\prime \prime}, S$ the closed unit ball of $E$ and $E^{\prime \prime}$ and the unit sphere of $E$, respectively, and we will write, for each $n \in \mathbf{N}, B_{n}=n B, B_{n}^{\prime \prime}=n B^{\prime \prime}$ and $S_{n}=n S$.

\section{The bw topology and local convexity.}

LEMMA 3.1. Let $E$ be a separable, non-reflexive Banach space. If $E$ contains no subspace isomorphic to $l^{1}$, there exists a subset $A$ of $E$ which is $b w$-closed but is not closed in the restriction to $E$ of the ew* topology on $E^{\prime \prime}$.

Proof. It is well known that for each $n \in \mathbf{N}$ the sphere $S_{n}$ is $\sigma\left(E^{\prime \prime}, E^{\prime}\right)$-dense in the closed ball $B_{n}^{\prime \prime}$. As $E$ is a separable Banach space that contains no subspace isomorphic to $l^{1}$ it follows from Rosenthal ([5], Theorem 3$)$ that $S_{n}$ is $\sigma\left(E^{\prime \prime}, E^{\prime}\right)$-sequentially dense in the ball $B_{n}^{\prime \prime}$, i.e. each $z \in B_{n}^{\prime \prime}$ can be approximated in $\sigma\left(E^{\prime \prime}, E^{\prime}\right)$ by a sequence contained in $S_{n}$. Hence if $\phi \in E^{\prime \prime} \backslash E$ and $\|\phi\|=1$, there exists for each $n \in \mathbf{N}$ a sequence $\left(x_{k, n}\right)_{k \in \mathrm{N}}$ contained in $S_{n}$ and converging to $n^{-1} \phi$ in $\sigma\left(E^{\prime \prime}, E^{\prime}\right)$.

We define $A=\left\{x_{k, n}: k, n \in \mathbf{N}\right\}$. For each $m \in \mathbf{N}$ we have

$$
\begin{aligned}
A \cap B_{m} & =\left\{x_{k, n}: k \in \mathbf{N}, n \leq m\right\} \\
& =\left(\left\{x_{k, n}: k \in \mathbf{N}, n \leq m\right\} \cup\left\{n^{-1} \phi: n \leq m\right\}\right) \cap B_{m},
\end{aligned}
$$

and since the set $\left\{x_{k, n}: k \in \mathbf{N}, n \leq m\right\} \cup\left\{n^{-1} \phi: n \leq m\right\}$ is $\sigma\left(E^{\prime \prime}, E^{\prime}\right)$ compact, it is $\sigma\left(E^{\prime \prime}, E^{\prime}\right)$-closed; then the set $A \cap B_{m}$ is closed in the restriction of $\sigma\left(E^{\prime \prime}, E^{\prime}\right)$ to $B_{m}$, but this topology is the same as the restriction of $\sigma\left(E, E^{\prime}\right)$ to $B_{m}$. This proves that $A$ is bw-closed.

On the other hand, let $U$ be a neighborhood of 0 in the ew* topology; there exists $W$, ew*-neighborhood of 0 such that $W+W \subset U$, and as $W$ is absorbent, there exists $n_{0} \in \mathbf{N}$ such that $n_{0}^{-1} \phi \in W$. By the definition of ew* topology we know there exists a $V, \sigma\left(E^{\prime \prime}, E^{\prime}\right)$-neighborhood of 0 satisfying

$$
W \cap B_{2 n_{0}}^{\prime \prime}=V \cap B_{2 n_{0}}^{\prime \prime}
$$

As $\left(x_{k, n_{0}}\right)_{k \in \mathrm{N}}$ converges to $n^{-1} \phi$ in the $\sigma\left(E^{\prime \prime}, E^{\prime}\right)$-topology there exists $k_{0} \in \mathbf{N}$ such that $x_{k_{0}, n_{0}}-n_{0}^{-1} \phi \in V$ and then

$$
x_{k_{0}, n_{0}}=x_{k_{0}, n_{0}}-n_{0}^{-1} \phi+n_{0}^{-1} \phi \in\left(V \cap B_{2 n_{0}}^{\prime \prime}\right)+W \subset W+W \subset U .
$$

This proves that 0 belongs to the closure of $A$ in the ew* topology, and since $0 \in E, 0$ is in the closure of $A$ in the restriction of ew* to $E$ (we denote this topology rew*). Thus $A$ is not closed in rew*. 
Proposition 3.2. Let $E$ be a separable Banach space that contains no subspace isomorphic to $l^{\prime}$. The bw topology on $E$ is locally convex if and only if $E$ is reflexive.

Proof. If $E$ is reflexive we saw in (2.7) that bw is a locally convex topology. Conversely, if $E$ is not reflexive, (3.1) and (2.6) prove that the bw topology is not locally convex.

Lemma 3.3. Let $E$ be a Banach space and $F$ a closed linear subspace of $E$. The bw topology of $F$ is the restriction to $F$ of the bw topology on $E$.

Proof. We denote $\mathrm{bw}(E)$ and $\mathrm{bw}(F)$ the bw topology on $E$ and $F$ respectively. It is clear that the restriction of $\mathrm{bw}(E)$ to $F$ is coarser than $\mathrm{bw}(F)$.

On the other hand, if $U$ is bw( $F)$-open, let $V$ be the union of $U$ and $E \backslash F$. It is sufficient to prove that $V$ is $\operatorname{bw}(E)$-open. If $B$ is a bounded subset of $E$, as $\sigma\left(F, F^{\prime}\right)$ coincides with the restriction of $\sigma\left(E, E^{\prime}\right)$ to $F$, there exists $W, \sigma\left(E, E^{\prime}\right)$-open, such that

$$
U \cap B=U \cap(B \cap F)=(W \cap F) \cap(B \cap F)=(W \cap F) \cap B,
$$

and then

$$
V \cap B=(W \cup(E \backslash F)) \cap B,
$$

and since $W \cup(E \backslash F)$ is $\sigma\left(E, E^{\prime}\right)$-open, $V$ is bw( $\left.E\right)$-open.

Proposition 3.4. Let $E$ be a Banach space that contains no subspace isomorphic to $l^{1}$. The bw topology on $E$ is locally convex if and only if $E$ is reflexive.

Proof. If $E$ is not reflexive, there exists a separable nonreflexive subspace $F$ of $E$. Obviously $F$ contains no subspace isomorphic to $l^{\prime}$. From (3.2) it follows that the bw topology on $F$ is not locally convex and then (3.3) shows that bw is not a locally convex topology on $E$. This fact and (2.7) prove the theorem.

Lemma 3.5. There exists a subset $A$ of $l^{1}$ which is bw-closed but is not closed in the restriction to $l^{1}$ of the ew*-topology of $\left(l^{1}\right)^{\prime \prime}$.

Proof. For each $n \in \mathbf{N}$, we denote by $e_{n}$ the sequence of $l^{1}(0,0, \ldots, 1,0, \ldots)$ where the one is in the $n$th place. 
Let $A_{0}$ be the set $A_{0}=\left\{e_{n}: n \in \mathbf{N}\right\}$. For each $n \in \mathbf{N}, e_{n}$ is a $\sigma\left(l^{1}, l^{\infty}\right)$-isolated point of $A_{0}$, and then $A_{0}$ is a $\sigma\left(l^{1}, l^{\infty}\right)$-closed set which is not $\sigma\left(l^{1}, l^{\infty}\right)$-compact. Consequently $A_{0}$ is not $\sigma\left(\left(l^{1}\right)^{\prime \prime}, l^{\infty}\right)$-closed. Thus there exists a linear form $\phi$ that belongs to the $\sigma\left(\left(l^{1}\right)^{\prime \prime}, l^{\infty}\right)$-closure of $A_{0}$ and $\phi \notin A_{0}$. Obviously $\|\phi\| \leq 1$ and $\phi \in\left(l^{1}\right)^{\prime \prime} \backslash l^{1}$.

Now, for each $n \in \mathbf{N}$, we define

$$
A_{n}=\left\{n\left(e_{p}-e_{q}\right)+e_{k} / n: p, q, k \in \mathbf{N}, p \neq q, p<k, q<k\right\} .
$$

$A_{n}$ is contained in the sphere of radius $2 n+1 / n$ of $l^{1}$ and if $n \in \mathbf{N}$, $n \geq 2$, it is not hard to check that $A_{n}$ is $\sigma\left(l^{1}, l^{\infty}\right)$-closed.

Let $V$ be a balanced, convex $\sigma\left(\left(l^{1}\right)^{\prime \prime}, l^{\infty}\right)$-neighborhood of 0 . As $\phi$ is an accumulation point of $A_{0}, \phi+V / 3 n$ contains an infinite number of points of $A_{0}$. If $e_{p}, e_{q}, e_{k} \in(\phi+V / 3 n) \cap A_{0}$ with $p<q<k$, we have

$$
\begin{aligned}
{\left[n\left(e_{p}-e_{q}\right)+\frac{1}{n} e_{k}\right]-\frac{1}{n} \phi } & =n\left(e_{p}-\phi\right)+n\left(\phi-e_{q}\right)+\frac{1}{n}\left(e_{k}-\phi\right) \\
& \in \frac{1}{3} V+\frac{1}{3} V+\frac{1}{3 n^{2}} V \subset V .
\end{aligned}
$$

Thus $(\phi / n+V) \cap A_{n}$ is a nonempty set. This proves that $\phi / n$ belongs to the $\sigma\left(\left(l^{1}\right)^{\prime \prime}, l^{\infty}\right)$-closure of $A_{n}$.

Now, we define $A=\cup_{n=2}^{\infty} A_{n}$. For each $m \in \mathbf{N}$, we have

$$
A \cap B_{m}=\bigcup\left\{A_{n}: n \in \mathbf{N}, 5 \leq 2 n^{2}+1 \leq m n\right\} .
$$

This set is obviously $\sigma\left(l^{1}, l^{\infty}\right)$-closed, and thus $A$ is a bw-closed set.

On the other hand, as for each $n \in \mathbf{N}, \phi / n$ belongs to the $\sigma\left(\left(l^{1}\right)^{\prime \prime}, l^{\infty}\right)$-closure of $A_{n}$; reasoning as in the last part of the proof of Lemma 3.1 proves that 0 belongs to the closure on ew* of $A$, and as 0 does not belong to $A$, we see that $A$ is not closed in the topology restriction to $l^{1}$ of ew* on $\left(l^{1}\right)^{\prime \prime}$.

Proposition 3.6. Let $E$ be a Banach space that contains a subspace isomorphic to $l^{1}$. Then $b w$ is not a locally convex topology on $E$.

Proof. From (3.5) we get that the bw topology on $l^{1}$ is not locally convex. Hence if $E$ contains a subspace isomorphic to $l^{1}$, Lemma 3.3 and the conservation of bw topologies under isomorphisms prove that bw is not a locally convex topology on $E$.

THEOREM 3.7. Let $E$ be a Banach space. $b w$ is a locally convex topology on $E$ if and only if $E$ is reflexive. 
Proof. If $E$ is reflexive, (2.7) gives us the result. Conversely, if bw is a locally convex topology on $E$, from (3.6) it follows that $E$ contains no subspace isomorphic to $l^{1}$ and (3.4) shows that $E$ must be reflexive.

\section{REFERENCES}

[1] H. S. Collins, Completeness and compactness in linear topological spaces, Trans. Amer. Math. Soc., 79 (1955), 256-280.

[2] M. M. Day, Normed Linear Spaces, Berlin 1962.

[3] J. Ferrera, Espacios de funciones debilmente continuas sobre espacios de Banach, Tesis Doctoral, Universidad Complutense, Madrid (1980).

[4] J. Horvath, Topological Vector Spaces and Distributions, Addison-Wesley, Reading, Massachusetts, 1959.

[5] H. P. Rosenthal, Some recent discoveries in the isomorphic theory of Banach Spaces, Bull. Amer. Math. Soc., 84 (1978), 803-831.

[6] H. H. Schaefer, Topological Vector Spaces, Springer-Verlag, Berlin and New York, 1971.

[7] R. F. Wheeler, The equicontinuous weak* topology and semi-reflexivity, Studia Mathematica, XLI (1972), 243-256.

Received December 22, 1981.

Facultad de Mathematicas

UNIVERSIDAD COMPLUTENSE

MADRID-3 (SPAIN) 


\section{PACIFIC JOURNAL OF MATHEMATICS \\ EDITORS}

DONALD BABBITT (Managing Editor)

University of California

Los Angeles, CA 90024

Hugo RossI

University of Utah

Salt Lake City, UT 84112

C. C. MOOre and ARThur OGus

University of California

Berkeley, CA 94720
J. Dugundi

Department of Mathematics

University of Southern California

Los Angeles, CA 90089-1113

R. FINN and H. SAMELSON

Stanford University

Stanford, CA 94305

ASSOCIATE EDITORS

$\begin{array}{lllll}\text { R. Arens } & \text { E. F. Beckenbach } & \text { B. H. Neumann } & \text { F. Wolf } & \text { K. Yoshida }\end{array}$ $(1906-1982)$

\section{SUPPORTING INSTITUTIONS}

UNIVERSITY OF ARIZONA

UNIVERSITY OF BRITISH COLUMBIA

CALIFORNIA INSTITUTE OF TECHNOLOGY

UNIVERSITY OF CALIFORNIA

MONTANA STATE UNIVERSITY

UNIVERSITY OF NEVADA, RENO

NEW MEXICO STATE UNIVERSITY

OREGON STATE UNIVERSITY
UNIVERSITY OF OREGON

UNIVERSITY OF SOUTHERN CALIFORNIA

STANFORD UNIVERSITY

UNIVERSITY OF HAWAII

UNIVERSITY OF TOKYO

UNIVERSITY OF UTAH

WASHINGTON STATE UNIVERSITY

UNIVERSITY OF WASHINGTON 


\section{Pacific Journal of Mathematics}

\section{Vol. 110, No. $1 \quad$ September, 1984}

Wojciech Abramczuk, A class of surjective convolution operators $\ldots \ldots \ldots \ldots 1$

K. Adachi, Extending bounded holomorphic functions from certain

subvarieties of a weakly pseudoconvex domain $\ldots \ldots \ldots \ldots \ldots \ldots$

Malvina Florica Baica, An algorithm in a complex field and its application

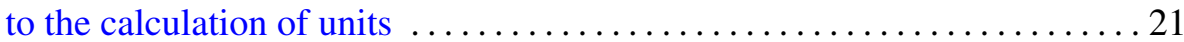

Giuliana Bianchi and Robert Cori, Colorings of hypermaps and a

conjecture of Brenner and Lyndon $\ldots \ldots \ldots \ldots \ldots \ldots \ldots \ldots \ldots \ldots \ldots \ldots$

Ronald James Evans, Determinations of Jacobsthal sums . . .......... 49

Leslie Foged, Characterizations of $\aleph$-spaces .................... 59

Nassif A. Ghoussoub and Paulette Saab, Weak compactness in spaces of

Bochner integrable functions and the Radon-Nikodým property . . . . . . 65

J. Gómez Gil, On local convexity of bounded weak topologies on Banach

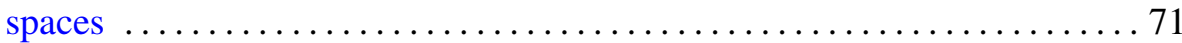

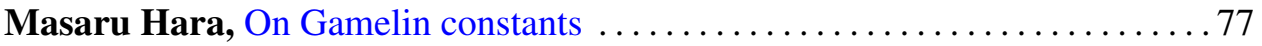

Wilfried Hauenschild, Eberhard Kaniuth and Ajay Kumar, Harmonic

analysis on central hypergroups and induced representations $\ldots \ldots \ldots 83$

Eugenio Hernandez, An interpolation theorem for analytic families of

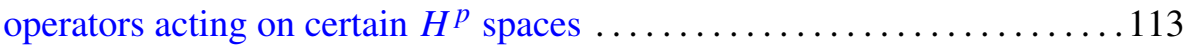

Thomas Alan Keagy, On "Tauberian theorems via block-dominated

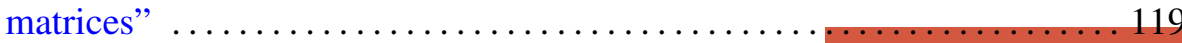

Thomas Landes, Permanence properties of normal structure $\ldots \ldots \ldots \ldots \ldots 125$

Daniel Henry Luecking, Closed ranged restriction operators on weighted

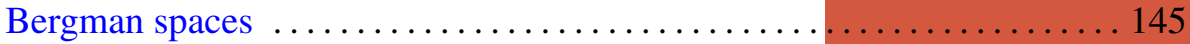

Albert Thomas Lundell, The $p$-equivalence of $\mathrm{SO}(2 n+1)$ and $\mathrm{Sp}(n) \ldots \ldots 161$

Mark D. Meyerson, Remarks on Fenn's "the table theorem" and Zaks' "the chair theorem" ..................................... 167

Marvin Victor Mielke, Homotopically trivial toposes . . . . . . . . . . 171

Gerard J. Murphy, Hyperinvariant subspaces and the topology on Lat A . . 183

Subhashis Nag, On the holomorphy of maps from a complex to a real manifold

Edgar Milan Palmer and Robert William Robinson, Enumeration of self-dual configurations ................................. 203

John J. Walsh and David Clifford Wilson, Continuous decompositions

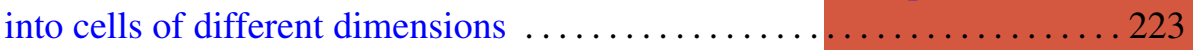

Walter John Whiteley, Infinitesimal motions of a bipartite framework .....233 